$\mathrm{DE}$

M E D I C I N A

T R O P I C A L

$\mathrm{DE}$

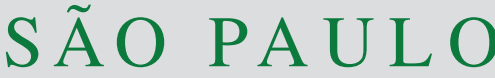

JOURNAL OF THE SÃO PAULO INSTITUTE OF TROPICAL MEDICINE

1 Universidade de São Paulo, Faculdade de Medicina, Hospital das Clínicas, LIM 03, São Paulo, São Paulo, Brazil

${ }^{2}$ Blood Systems Research Institute, San Francisco, California, USA

3Universidade Estadual de Montes Claros, Montes Claros, Minas Gerais, Brazil

${ }^{4}$ Hospital Sírio-Libanês, Instituto de Ensino e Pesquisa, São Paulo, São Paulo, Brazil

${ }^{5}$ Universidade de São Paulo, Instituto de Medicina Tropical de São Paulo, São Paulo, São Paulo, Brazil

${ }^{6}$ Universidade Federal de São João del-Rei, São João del-Rei, Minas Gerais, Brazil

${ }^{7}$ Instituto de Infectologia Emílio Ribas, São Paulo, São Paulo, Brazil

${ }^{8}$ Universidade Federal do ABC, São Paulo, São Paulo, Brazil

'Universidade Federal de Minas Gerais, Faculdade de Medicina, Hospital das Clínicas, Belo Horizonte, Minas Gerais, Brazil

Correspondence to: Ester Cerdeira Sabino Universidade de São Paulo, Instituto de Medicina Tropical de São Paulo, Avenida Dr. Enéas Carvalho de Aguiar, 470, CEP 05403-000, São Paulo, SP, Brazil Tel: +551130617041

E-mail: sabinoec@gmail.com

Received: 11 October 2018

Accepted: 2 January 2019

\section{Lack of evidence of seronegative infection in an endemic area of Chagas disease}

\author{
Léa Campos de Oliveira1, Tzong-Hae Lee ${ }^{2}$, Ariela Mota Ferreira ${ }^{3}$, Ana Luiza \\ Bierrenbach $^{4}$, Marcela de Souza-Basqueira ${ }^{5}$, Cláudia Di Lorenzo Oliveira ${ }^{6}$, \\ Clareci Silva Cardoso ${ }^{6}$, Carlos Henrique Valente Moreira ${ }^{5,7}$, Marcio K. \\ Oikawa $^{8}$, Antonio Luiz P. Ribeiro' ${ }^{9}$, Michael P Busch ${ }^{2}$, Ester Cerdeira Sabino ${ }^{5}$
}

\section{ABSTRACT}

The diagnosis of Chagas disease is based on the detection of Trypanosoma cruzi ( $T$. cruzi)-specific antibodies. Nonetheless, there is concern about the sensitivity of current serological assays due to reports of $T$. cruzi PCR positivity among seronegative individuals. The aim of this study was to evaluate if $T$. cruzi seronegative infections occur in endemic areas. We recruited 2,157 individuals that were identified as having Chagas disease in a public health system database of an endemic region in Brazil. All participants were interviewed and 2,091 had a sample collected for serological and PCR testing. From these, $149(7.1 \%)$ had negative serological results. PCR was positive in 610 samples $(31.4 \%)$ of the 1,942 seropositive samples but in none of the 149 samples from seronegative participants. True $T$. cruzi seronegative infections seem to be rare (95\% CI 0-3.7) and should not be a concern for blood supply, which relies on antibody screening.

KEYWORDS: Chagas disease. Diagnosis. Infectious diseases. Prevention and control.

\section{INTRODUCTION}

Chagas disease is caused by the parasite Trypanosoma cruzi ${ }^{1}$. In recent years, despite the progressive decline of the estimated incidence and prevalence of Chagas disease, it remains one of the world's most neglected diseases, with an estimated 5.7 million infected people mostly in 21 resource-limited countries in America ${ }^{2}$.

The diagnosis is based on serological assays because parasitemia is in general low or undectectable during the chronic phase of the infection. The earliest tests developed for blood screening in Latin America had low sensitivity and reproducibility ${ }^{3}$. Consequently, for many years, countries like Brazil required the use of parallel serological tests for T. cruzi blood screening ${ }^{4}$.

Low reacting samples may not be detectable by all serological assays. This situation is commonly identified by donor screening in Latin American countries and in the US and these donations could be missed by some assays and represent a threat to blood supply ${ }^{5-8}$. In this context, it is reassuring that parasitemia is rarely detected by sensitive PCR tests performed on DNA derived from large volumes of blood samples from donors with low antibody titers, suggesting that they may represent resolved infections with waning antibodies ${ }^{9}$.

Another possible threat is the presence of so-called "serosilent infections", in which parasitemia is detectable in seronegative individuals ${ }^{10-12}$. Rare cases of "serosilent infection" were previously described for HIV and HCV and, in general, they are 
related to individuals with poor immune response ${ }^{13}$. In a previous study, we evaluated the frequency of seronegative infections by testing 500 seronegative blood donors from endemic regions in Brazil by a sensitive PCR testing ${ }^{14}$.

In the present study, to further investigate the frequency of seronegative T. cruzi infections, we performed a sensitive, high-volume input $T$. cruzi PCR assay on coded samples from 2,091 individuals with cardiac abnormalities from a region in Brazil with a high prevalence of Chagas Disease. We found 149 (7\%) seronegative individuals but none of them tested positive by PCR, showing that if seronegative parasitemic infections exist, they are very rare.

\section{METHODS}

\section{Study design}

This study is part of the Sao Paulo-Minas Gerais Tropical Medicine Research Center (SaMi-Trop), a prospective cohort of patients with Chagas disease ${ }^{15}$. Selection of patients was made by using the database of the Telehealth Network of Minas Gerais, a program designed to support primary care in Minas Gerais State that collects and analyses patients' ECG and clinical data ${ }^{16}$. Patients living within a limited region in the Northern part of Minas Gerais State that has a high prevalence of $T$. cruzi infection were included if they had ECG abnormalities and self-reported Chagas Disease. From 4,689 eligible patients, 2,157 individuals were recruited, interviewed and submitted to ECG and sample collection. From these, we obtained blood samples and performed serology and PCR in 2,091 individuals, which were included in this study. All these subjects signed the informed consent for additional testing including PCR. This study was approved by National Council Research Ethics - CONEP (Certificate of presentation for Ethical Appreciation CAAEE N ${ }^{\circ}$ 00580612.8.0000.0065).

\section{Blood processing}

At the time of the enrollment interview, $8 \mathrm{~mL}$ of peripheral blood were collected in serum separator tubes (SST) for serological analyses and $12 \mathrm{~mL}$ of ethylenediaminetetraacetic acid (EDTA)-anticoagulated blood were collected and immediately mixed with an equal volume of $6 \mathrm{M}$ guanidine/HCl-0.2M EDTA solution for PCR. These samples were aliquoted and frozen in Brazil at $-20^{\circ} \mathrm{C}$. Aliquots of guanidine-lysed blood samples were shipped to the Blood Systems Research Institute (San Francisco, CA, USA) on dry ice, followed by maintenance at $-70{ }^{\circ} \mathrm{C}$. All testing was performed on coded samples.

\section{Serology testing}

All samples were initially screened using the chemiluminescent microparticle immunoassay (ChIA) method for detection of antibodies to T. cruzi (Architect Chagas, Abbott Laboratories, Wiesbaden Germany). Samples with negative results were retested with two other enzyme immunoassays (EIAs: Chagatest v.4, Wiener and Chagas, Diasorin). We classified ChIA negative samples as inconclusive when they were reactive on one or both of the antibody assays used for retesting.

\section{PCR procedure}

The target-capture (TC) real-time (RT) PCR assay used in this study was developed based on the PCR method described by Pyron et al. ${ }^{17}$, that targets satellite T. cruzi DNA. The DNA extraction was improved through the use of a TC step that employed magnetic beads coated with a T. cruzi-specific 20-mer capture oligonucleotide ${ }^{9}$.

Two replicate TC-PCR assays were performed and results were interpreted as positive if both replicates were positive. If only one replicate result was positive, the sample was considered as PCR inconclusive, and another aliquot of this sample was processed in four replicates. The results were then considered positive if at least two of the four additional replicates were positive. Quantification of parasitemia was based on a standard curve derived from cultured parasites spiked into whole blood prior to lysis, with a detection range of 0.1 to 10,000 parasites $/ \mathrm{mL}^{9,14}$

\section{Statistical analysis}

Data are presented as means and standard deviations or as numbers and percentages. Shapiro-Wilk tests were used to verify data normality. ANOVA tests were used for comparison of antibodies levels with parasitemia. Chisquare tests and/or Fisher exact tests were used to compare categorical variables between groups and Kruskall-Wallis tests were used to compare continuous variables between groups. A value of $\mathrm{p}<0.05$ was considered significant.

\section{RESULTS}

The EIA and PCR results obtained by testing 2,091 coded samples are summarized in Figure 1. One-hundred fifty-eight patients' samples had negative results by the Architect ChIA antibody test (ARCHITECT Chagas Abbott, Illinois USA). From these, nine had reactive results with at least one of the other two EIA assays (Kit Chagatest - ELISA recombinant v.4.0 -Wiener, Rosario, 
Argentine and Kit Test ELISA Chagas III - (Grupo Bios S.A - Diasorin, Santiago, Chile) and were classified as serologically inconclusive.

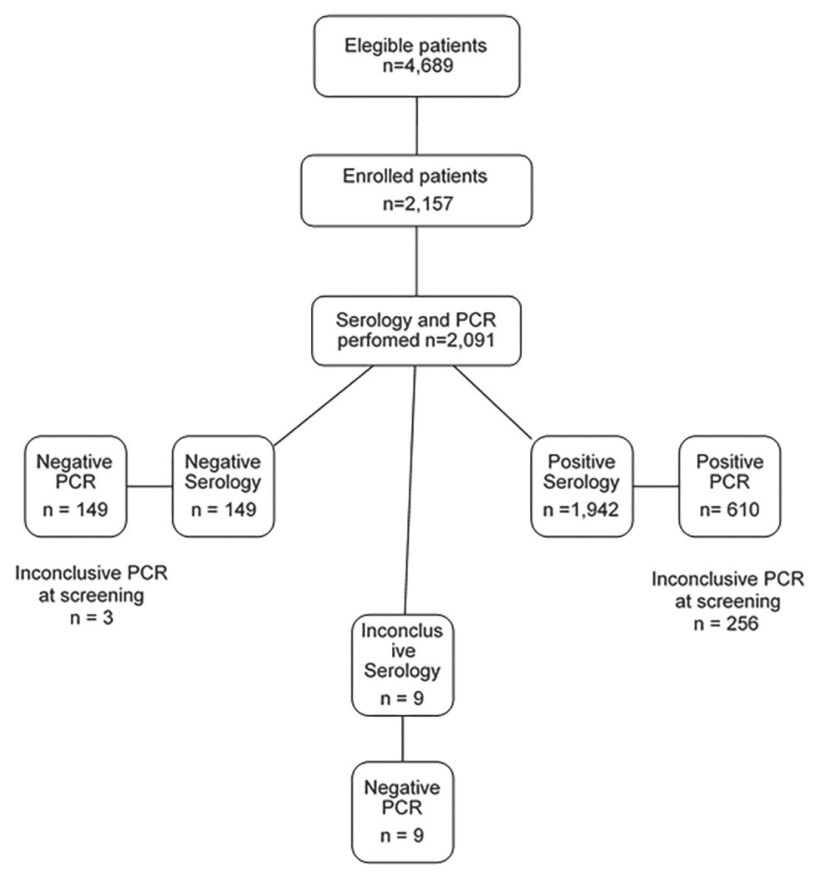

Figure 1 - Serological and PCR results of studied patients.

None of the 158 coded samples with negative or inconclusive ChIA/EIA results were positive by PCR. In contrast, 610 (31.4\%) of the 1,942 ChIA seropositive samples were PCR positive, $(\mathrm{p}<0.001$ for comparison with the EIA-negative samples). The concentration of parasites detected by PCR ranged from 0.1 to 667 parasites $/ \mathrm{mL}$.

Table 1 summarizes the epidemiologic and clinical characteristics of patients, as well as PCR results, of seropositive and seronegative participants. Of the 149 seronegative individuals, $110(74 \%)$ reported a previous Chagas diagnosis during the study interview, 64 (43\%) referred at least one relative with a diagnosis of Chagas disease, and 26 indicated that their mothers were $T$. cruzi positive. Only nine participants stated that they had previously received benznidazole (BZN) treatment. Given that we have screened 2,091 individuals, we can state that the prevalence of seronegative infection in the population may vary from 0 to 3.7 , with a $95 \%$ confidence interval.

\section{DISCUSSION}

In this study, we could not find evidence of T. cruzi seronegative infection after rigorous serological and PCR testing of coded samples from 2,091 individuals that disclosed Chagas disease in their clinical histories and presenting ECG test abnormalities in the primary care center. Seronegative results using a combination of sensitive antibody assays were found in $7 \%$ of the patients, but none of them tested positive by PCR for T. cruzi. PCR was positive in only $31.4 \%$ of the coded samples from seropositive individuals.

Previous reports have reported seronegative T. cruzi infections, raising concerns on the sensitivity of serological assays for diagnostics and blood bank donor screening. Salomone et al. ${ }^{12}$ evaluated 194 subjects from endemic regions, including 110 sent from a cardiology clinic. In this sample, 80 patients were negative by the three serological tests used (indirect-hemagglutination, immunofluorescence and ELISA), and of those, $12(15 \%)$ were positive by PCR. In that study, PCR was positive in 24 of 114 (21\%) seropositive patients. Of note, no control group derived from a low risk population was tested to determine false positive rates of the PCR assay. Gomes et al. ${ }^{11}$, tested 100 T. cruzi-infected individuals from highly endemic areas by hemagglutination, EIA and PCR. PCR was positive in 66 $(84 \%)$ of the 79 seropositive samples and in $10(48 \%)$ of 21 seronegative individuals. They also evaluated samples from 13 seronegative controls that were not from endemic

Table 1 - Epidemiological and clinical characteristics and PCR testing results of Chagas disease patients from endemic areas in Minas Gerais State, highlighting negative versus positive serological results.

\begin{tabular}{lccc}
\hline Variable & $\begin{array}{c}\text { Seronegative individuals } \\
\mathrm{n}=149(\%)\end{array}$ & $\begin{array}{c}\text { Seropositive individuals } \\
\mathrm{n}=1942(\%)\end{array}$ & P-value \\
\hline Age mean \pm SD, & $63 \pm 14.4$ & $59 \pm 12.7$ & $<0.001^{\mathrm{a}}$ \\
Male & $63(42.3)$ & $632(32.5)$ & $0.015^{\mathrm{b}}$ \\
Mother with Chagas disease & $26(17.4)$ & $369(19)$ & $0.641^{\mathrm{b}}$ \\
Other family member with Chagas disease & $64(42.9)$ & $1,002(51.6)$ & $0.042^{\mathrm{b}}$ \\
Self-reported Chagas disease & $110(73.8)$ & $1,870(96.3)$ & $<0.001^{\mathrm{b}}$ \\
Benznidazole treatment & $9(6.0)$ & $492(25.3)$ & $<0.001^{\mathrm{b}}$ \\
Positive PCR assay & $0(0)$ & $610(31.4)$ & $<0.001^{\mathrm{c}}$ \\
\hline
\end{tabular}

${ }^{\mathrm{a}}$ Mann Whitney test; ${ }^{\mathrm{b}}$ Chi-square test; ${ }^{\mathrm{c}}$ Fisher exact test. 
areas, and all of them were PCR negative. More recently, Batista et al..$^{10}$ evaluated 41 seronegative and serologically inconclusive cases with mega-esophagus, and reported 31 (76\%) as PCR positive. In this study PCR was positive in $42(93 \%)$ of the 45 seropositive individuals, and in none of the 18 seronegative controls.

Different factors must be considered when comparing serology and PCR results. Serological methods have improved over the years as a consequence of the use of recombinant proteins. Nowadays, the main problem seems to be cross-reactions with other diseases, especially leishmaniasis. However, PCR results still show high variability, depending on $T$. cruzi lineages and amplified target regions. Another important issue is that parasitemia fluctuates in patients with chronic Chagas disease over the prolonged course of infection ${ }^{18}$.

It is important to emphasize that our study has limitations. Here, 492 (25\%) of the seropositive individuals had been previously treated with BZN. Our studied population was older than the patients in other studies such as the Benefit trial that yielded a higher rate of PCR positivity $(66.2 \%)^{19}$. Nevertheless, if the prevalence of "serosilent" infection in the study population was $1 \%$, we would have had approximately 20 "serosilent" individuals within the 149 ones tested as seronegative. Given the $31.3 \%$ of detection rate of $T$. cruzi DNA by our TC-PCR assay in coded samples from this cohort seropositive participants, the PCR test would have detected six cases, but none was observed. In fact, we can also state with $95 \%$ CI that the prevalence is below $3.7 \%$.

Some of these factors may account for the fact that the above-mentioned studies detected "serosilent" infections, while this study and the previous one performed by our group on blood donor samples did not. The seronegative individuals in our study were older and hence may have resolved infections and seroreverted. We used sensitive blood bank ChIA and EIAs, which are currently used for screening and diagnosis, while the other studies that reported "serosilent" infections used less sensitive assays such as hemaglutination, immunofluorescence or earlier versions of EIAs. Moreover, given the natural history of Chagas disease, i.e. the long incubation period before symptoms develop, and the difficulty of tracking and associating donors with recipients long after a transfusion event, it is reassuring that no unambiguous case report of transfusion-transmission of Chagas from a seronegative infected individual has taken place, even if this type of transmission does actually occur.

In conclusion, our data on the absence of seronegative infections in a large cohort of high-risk clinical cases confirm our previous results from a retrospective Brazilian blood donor study. These findings are reassuring and indicate that donor serological screening tests are highly sensitive in detecting antibodies against $T$. cruzi.

\section{FUNDING}

This study was supported by the National Institute of Allergy And Infectious Diseases of the National Institutes of Health (grant $\mathrm{N}^{\circ}$ P50AI098461) and by the Brazilian National Research Council CNPq (grant $\mathrm{N}^{\circ}$ 467043/2014-0). ALPR is also supported by personal grants from CNPq and FAPEMIG, the State of Minas Gerais Research Agency.

\section{AUTHORS' CONTRIBUTIONS}

The authors' contributions were: substantial contributions to the conception or design of the work; or the acquisition, analysis, or interpretation of data for the work: LCO, ECS, ALPR, ALB, CLO, CHVM, MKO, MSB. Drafting the work or revising it critically for important intellectual content: LCO, ALB, CLO. Final approval of the version to be published: all. All authors read and approved the final manuscript.

\section{REFERENCES}

1. Bern C. Chagas' Disease. N Engl J Med. 2015;373:456-66.

2. Ribeiro AL, Nunes MP, Teixeira MM, Rocha MO. Diagnosis and management of Chagas disease and cardiomyopathy. Nat Rev Cardiol. 2012;9:576-89.

3. Camargo ME, Segura EL, Kagan IG, Souza JM, Carvalheiro JR, Yanovsky JF, et al. Three years of collaboration on the standardization of Chagas' disease serodiagnosis in the Americas: an appraisal. Bull Pan Am Health Organ. 1986;20:233-44.

4. Salles NA, Sabino EC, Cliquet MG, Eluf-Neto J, Mayer A, Almeida-Neto C, et al. Risk of exposure to Chagas' disease among seroreactive Brazilian blood donors. Transfusion. 1996;36:969-73.

5. Custer B, Agapova M, Bruhn R, Cusick R, Kamel H, Tomasulo $\mathrm{P}$, et al. Epidemiologic and laboratory findings from 3 years of testing United States blood donors for Trypanosoma cruzi. Transfusion. 2012;52:1901-11.

6. Otani MM, Vinelli E, Kirchhoff LV, del Pozo A, Sands A, Vercauteren G, et al. WHO comparative evaluation of serologic assays for Chagas disease. Transfusion. 2009;49:1076-82.

7. Remesar M, Sabino EC, del Pozo A, Mayer A, Busch MP, Custer B. Bimodal distribution of Trypanosoma cruzi antibody levels in blood donors from a highly endemic area of Argentina: what is the significance of low-reactive samples? Transfusion. 2015;55:2499-504. 
8. Sabino EC, Salles NA, Sarr M, Barreto AM, Oikawa M, Oliveira $\mathrm{CD}$, et al. Enhanced classification of Chagas serologic results and epidemiologic characteristics of seropositive donors at three large blood centers in Brazil. Transfusion. 2010;50:2628 37.

9. Sabino EC, Lee TH, Montalvo L, Nguyen ML, Leiby DA, Carrick DM, et al. Antibody levels correlate with detection of Trypanosoma cruzi DNA by sensitive polymerase chain reaction assays in seropositive blood donors and possible resolution of infection over time. Transfusion. 2013;53:125765.

10. Batista AM, Aguiar C, Almeida EA, Guariento ME, Wanderley JS, Costa SC. Evidence of Chagas disease in seronegative Brazilian patients with megaesophagus. Int J Infect Dis. 2010;14:e974-7.

11. Gomes ML, Galvão LM, Macedo AM, Pena SD, Chiari E. Chagas' disease diagnosis: comparative analysis of parasitologic, molecular, and serologic methods. Am J Trop Med Hyg. 1999;60:205-10.

12. Salomone OA, Basquiera AL, Sembaj A, Aguerri AM, Reyes ME, Omelianuk M, et al. Trypanosoma cruzi in persons without serologic evidence of disease, Argentina. Emerg Infect Dis. 2003;9:1558-62.

13. Spivak AM, Sydnor ER, Blankson JN, Gallant JE. Seronegative HIV-1 infection: a review of the literature. AIDS. 2010;24:140714.
14. Sabino EC, Ribeiro AL, Salemi VM, Di Lorenzo Oliveira C, Antunes AP, Menezes MM, et al. Ten-year incidence of Chagas cardiomyopathy among asymptomatic Trypanosoma cruziseropositive former blood donors. Circulation. 2013;127:110515.

15. Cardoso CS, Sabino EC, Oliveira CD, de Oliveira LC, Ferreira AM, Cunha-Neto E, et al. Longitudinal study of patients with chronic Chagas cardiomyopathy in Brazil (SaMi-Trop project): a cohort profile. BMJ Open. 2016;6:e11181.

16. Alkmim MB, Figueira RM, Marcolino MS, Cardoso CS, Pena de Abreu M, Cunha LR, et al. Improving patient access to specialized health care: the Telehealth Network of Minas Gerais, Brazil. Bull World Health Organ. 2012;90:373-8.

17. Piron M, Fisa R, Casamitjana N, López-Chejade P, Puig L, Vergés M, et al. Development of a real-time PCR assay for Trypanosoma cruzi detection in blood samples. Acta Trop. 2007;103:195-200.

18. Schijman AG. Molecular diagnosis of Trypanosoma cruzi. Acta Trop. 2018;184:59-66.

19. Morillo CA, Marin-Neto JA, Avezum A, Sosa-Estani S, Rassi A, Jr., Rosas F, et al. Randomized trial of Benznidazole for chronic Chagas' cardiomyopathy. N Engl J Med. 2015;373:1295-306. 